\title{
Análise mecânica de pilares mistos bambu-concreto
}

\author{
Humberto C. Lima Júnior ${ }^{1}$, Fábio L. Willrich², Gilmar Fabro ${ }^{3}$, Maxer A. Rosa², Leandro Tanabe² \& René B. G. Sabino ${ }^{1}$
}

\section{RESU MO}

0 objetivo deste trabalho foi avaliar o comportamento de pilares de bambu da espécie Dendrocalamus giganteus com e sem o preenchimento interior de concreto. Foram ensaiados, a compressão axial, 18 pilares com comprimentos de 1 , 1,5 e $2 \mathrm{~m}$ e, para cada comprimento, tal como 6 pilares, sendo 3 mistos de bambu-concreto e 3 formados apenas por colmos de bambu. 0 s resultados foram analisados através da teoria da instabilidade de casca cilíndrica e das propriedades mecânicas dos materiais. Curvas força vs. deformação, teóricas e experimentais, são apresentadas e a influência do comprimento dos pilares sobre as últimas forças resistentes foi avaliada por meio de análise de variância. Os pilares mistos bambu-concreto apresentaram comportamento não-linear e, em média, suas últimas forças resistentes mostraram valor da ordem de $50 \%$ da obtida pela Teoria da Resistência dos Materiais; já os pilares formados apenas por colmos de bambu, indicaram comportamento linear e suas últimas forças diferiram apenas $5 \%$ das teóricas, calculadas pela Teoria da Resistência dos M ateriais. Verifica-se que a utilização de pilares de bambu sem preenchimento de concreto apresenta maior viabilidade estrutural e econômica, podendo ser utilizada em obras de pequeno porte e de baixo custo.

Palavras-chave: elemento estrutural, material alternativo, reforço não metálico, resistência a compressão

\section{Mechanical analysis of hybrid bamboo-concrete columns}

\begin{abstract}
This work aimed to investigate the mechanical behaviour of Dendrocalamus giganteus bamboo columns filled with concrete. Eighteen columns were tested under axial compression. The columns were 1, 1.5 and $2 \mathrm{~m}$ high and, for each height, 6 columns were studied, in which three were filled with concrete and three were made only with the bamboo culms. The results were analysed by the instability theory of cylindrical shell and by the mechanical properties of the material. Theoretical and experimental load versus strain curves are presented and the influence of column height on the columns load capacity were evaluated by variance analysis. The bamboo-concrete columns presented a non linear behaviour and showed an ultimate load 50\% lower than the theoretical loads calculated by the mechanical resistance of the materials. The columns made only with bamboo culms presented a linear behaviour and the differences between the theoretical and experimental load were less than $5 \%$. Finally, the columns made with bamboo culms showed great potential to be used as an alternative in low-income housing.
\end{abstract}

Key words: structural element, alternative material, non-metallic reinforcement, compressive strength

\footnotetext{
${ }^{1}$ Núcleo de Tecnologia/UFPE, BR 104, km 59, s/n, Zona Rural, Sítio Juriti, CEP 55002-970, Caruaru, PE. Fone: (81) 8603-6716. E-mail: humbertolima@ufpe.br; buen089@gmail.com

2 UNIO ESTE. Rua Universitária 2069, CEP 85814-110, Cascavel, PR. Fone: (45) 3220-3221. E-mail: flwillrich@unioeste.br; marosa@unioeste.br.

3 ITAIPU Binacional. Av. Tancredo Neves 6731, CP 1555, CEP 85856-000, Foz do Iguaçu, PR. Fone: (45) 3520-6220. E-mail: fabro@itaipu.gov.br
} 


\section{INTRODUÇÃO}

A população mundial enfrenta, atualmente, uma série de desafios relacionados ao uso sustentável dos recursos naturais disponíveis na terra. Observa-se, a partir da década de 1950, que pesquisas vêm apontando para um desequilíbrio bastante perigoso da utilização indiscriminada e dispendiosa desses recursos, o qual se agravou com a duplicação da população mundial e a quintuplicação da produção econômica global (Riaño et al., 2002).

A escassez dos recursos naturais, associada ao desenvolvimento de novas tecnologias, vem incentivando novas pesquisas com materiais ambientalmente corretos; neste contexto se insere o bambu, que é um material vegetal cujas propriedades mecânicas indicam grande potencial a ser explorado pela engenharia. Apesar das formidáveis propriedades físicas e mecânicas, o bambu tem sido pouco utilizado como material na indústria da construção civil no Brasil devido, basicamente, a três fatores: à sua configuração geométrica, que impossibilita a confecção de peças estruturais usuais, como vigas e pilares de seções transversais retangulares; à sua baixa resistência ao cisalhamento, que não permite a cravação de pinos, tornando inviável o projeto de estruturas de maior porte, onde há necessidade de emendas e ligações e à durabilidade (Lima Jr. \& Dias, 2001; Chung \& Yu, 2002; Beraldo \& Rivero, 2003; Albermani et al., 2007).

O bambu apresenta longos colmos ocos no interior, os quais são fechados a intervalos mais ou menos regulares por um diafragma nas regiões dos nós; suas paredes têm excelente resistência à tração e à compressão, comparáveis às mais nobres madeiras, além de apresentar baixo peso específico, da ordem de $8,5 \mathrm{kN} \mathrm{m}^{-3}$ e rápido crescimento (3 a 6 anos) (Lima Jr. et al., 2008; Yu et al., 2005). Constata-se na literatura que algumas espécies de bambu podem atingir resistências a compressão e tração superiores a 60 e $250 \mathrm{MPa}$, respectivamente, e módulo de elasticidade na compressão e tração 21 e 18 GPa, respectivamente, (Lima Jr. et al., 2008). Tais propriedades físicas e mecânicas fazem com que os colmos de bambu apresentem elevada relação entre a rigidez a flexão e seu peso específico, além de grande resistência a compressão.

Considerando o exposto no parágrafo anterior, pode-se associar os colmos de bambu aos pilares utilizados nas estruturas de madeira. No sudeste asiático é bastante comum a utilização de colmos de bambu para a construção de andaimes em edifícios que podem ultrapassar $20 \mathrm{~m}$ de altura, nos quais os pilares são constituídos por um ou mais colmos de bambu (Yu et al., 2003). Ao se analisar pilares mistos de açoconcreto, onde tubos de aço são preenchidos com concreto, verifica-se a possibilidade de associação dos colmos de bambu aos tubos de aço, desde que os diafragmas sejam extraídos.

O presente trabalho foi realizado com o objetivo de avaliar as características mecânicas de pilares de bambu para serem empregados em pequenas construções rurais. Aqui se apresentam os resultados de uma investigação teórico-experimental sobre a utilização de colmos de bambu Dendrocalamus giganteus como pilares; para tanto, 18 pilares de bambu foram ensaiados à compressão centrada e metade dos pilares teve seus colmos preenchidos com concreto; enfim, diferentes alturas de pilares foram estudadas.

\section{MATERIAL E MÉTODOS}

\section{Metodologia de análise dos pilares Estabilidade física}

A grande maioria das normas sobre dimensionamento de estruturas de concreto armado sugere que a capacidade de carga de pilares curtos pode ser calculada com base na teoria da resistência dos materiais. Dentro deste contexto e definidas as leis constitutivas dos materiais e a seção transversal do elemento estrutural de concreto armado, pode-se obter os esforços resistentes, para o que se utiliza um sistema local de coordenadas $(\xi, \eta, \zeta)$, que passa pelo centro de gravidade da seção transversal e a integração das tensões definidas para o concreto e para o bambu, em função das curvas tensão-deformação de cada material; assim, o esforço resistente pode ser descrito com base na Eq. 1:

na qual $\sigma_{\mathrm{c}}(\varepsilon)$ e $\sigma_{\mathrm{b}}(\varepsilon)$ são as tensões no concreto e no bambu,

$$
P_{\xi}=-\left(\int_{A_{c}} \sigma_{c}(\varepsilon) \cdot d A+\sum_{n} A_{b n} \cdot \sigma_{b}(\varepsilon)_{n}\right)
$$

respectivamente; $A_{c}$ é a área do concreto e $A_{b n}$ é a área de bambu.

Segundo Lima Jr. et al. (2005), a tensão no concreto pode ser calculada com base na Eq. 2:

em que $\mathrm{E}_{\mathrm{co}}$ é o módulo de elasticidade secante do concreto,

$$
\sigma_{\mathrm{c}}=\left[\frac{\left(\frac{\mathrm{E}_{\mathrm{c}}}{\mathrm{E}_{\mathrm{co}}}\right) \cdot\left(\frac{\varepsilon_{\mathrm{c}}}{\varepsilon_{\mathrm{co}}}\right)-\left(\frac{\varepsilon_{\mathrm{c}}}{\varepsilon_{\mathrm{co}}}\right)^{2}}{1+\left(\left(\frac{\mathrm{E}_{\mathrm{c}}}{\mathrm{E}_{\mathrm{co}}}\right)-2\right) \cdot\left(\frac{\varepsilon_{\mathrm{c}}}{\varepsilon_{\mathrm{co}}}\right)}\right] \cdot \mathrm{f}_{\mathrm{c}} \quad \varepsilon_{\mathrm{c}} \leq \varepsilon_{\mathrm{cu}}
$$

dado pela Eq. 3, $\mathrm{E}_{\mathrm{c}}$ é o módulo de elasticidade do concreto calculado pela Eq. 4, $\varepsilon_{\mathrm{cu}}$ é a deformação última do concreto, dada pela Eq. 5 e $\varepsilon_{\text {co }}$ corresponde à deformação relativa à máxima tensão do concreto e que pode ser calculada com base na Eq. 6:

sendo $\mathrm{f}_{\mathrm{cmo}}$ igual a $70 \mathrm{MPa}, \mathrm{f}_{\mathrm{c}}$ a resistência a compressão

$$
\begin{aligned}
& \mathrm{E}_{\mathrm{co}}=\frac{\mathrm{f}_{\mathrm{c}}}{\varepsilon_{\mathrm{co}}} \quad(\mathrm{MPa}) \\
& \mathrm{E}_{\mathrm{c}}=\alpha_{\mathrm{e}} \cdot \alpha_{\beta} \cdot\left[\mathrm{f}_{\mathrm{c}} / \mathrm{f}_{\mathrm{cmo}}\right]^{1 / 3} \quad(\mathrm{MPa}) \\
& \frac{\varepsilon_{\mathrm{cu}}}{\varepsilon_{\mathrm{co}}}=\frac{1}{2} \cdot\left(\frac{1}{2} \cdot \frac{\mathrm{E}_{\mathrm{c}}}{\mathrm{E}_{\mathrm{co}}}+1\right)+\sqrt{\frac{1}{4} \cdot\left(\frac{1}{2} \cdot \frac{\mathrm{E}_{\mathrm{c}}}{\mathrm{E}_{\mathrm{co}}}+1\right)^{2}-\frac{1}{2}} \\
& \varepsilon_{\mathrm{co}}=-0,0017-0,0010 \cdot\left(\frac{\mathrm{f}_{\mathrm{c}}}{\mathrm{f}_{\mathrm{cmo}}}\right)
\end{aligned}
$$

média do concreto, $\alpha_{\mathrm{e}}$ igual a $21500 \mathrm{MPa}$ e $\alpha_{\mathrm{b}}$ um coeficiente que depende do tipo de agregado graúdo utilizado no concreto e igual a 1,2 para o agregado basáltico.

O comportamento tensão de compressão vs. deformação do bambu, foi considerado elástico linear até a ruptura (Lima Jr. et al., 2005); assim, as tensões no colmo de bambu podem ser definidas com base na Eq. 7: 


$$
\sigma_{\mathrm{b}}=\mathrm{E}_{\mathrm{b}} \cdot \varepsilon_{\mathrm{b}} \quad \varepsilon_{\mathrm{b}} \leq \varepsilon_{\mathrm{br}}
$$

em que $E_{b}$ é o módulo de elasticidade do bambu, $\varepsilon_{\mathrm{b}}$ é a deformação no bambu e $\varepsilon_{\mathrm{br}}$ é a deformação de ruptura do bambu. $O$ valor de $\varepsilon_{\text {br }}$ foi determinado na primeira fase desta pesquisa, sendo seu valor igual 2,63\%o. Lima Jr. et al. (2008) mostraram que os módulos de elasticidade à compressão do bambu nas regiões nodal e internodal, com os respectivos desvios-padrão, são 21,88 \pm 4,57 GPa e 20,50 \pm 4,79 GPa, respectivamente.

\section{Estabilidade geométrica}

Todas as peças submetidas a compressão estão sujeitas à ruína por perda de estabilidade geométrica. No caso dos pilares mistos bambu-concreto podem ocorrer dois modos básicos: perda de estabilidade global e perda de estabilidade local. O primeiro caso, denominado flambagem global, pode ser avaliado diretamente com base na Teoria da Elasticidade, em que a força necessária para que os pilares percam a estabilidade é expressa pela Eq. 8:

$$
P_{c r}=\frac{\pi^{2} \cdot E \cdot I}{(\lambda \cdot L)^{2}}
$$

na qual E é o módulo de elasticidade do material que compõe o pilar, I é o momento de inércia da seção transversal, L é a altura do pilar medida entre os apoios e $\lambda$ é um coeficiente que depende das condições de contorno do pilar em que, para o caso engaste-rótula e engaste-engaste são, respectivamente, 0,7 e 0,5 . Salienta-se que para o caso dos colmos de bambu, onde existe a presença do desalinhamento do eixo longitudinal, o valor da carga crítica calculada pela Eq. 8 é reduzido.

No caso de cilindros de paredes finas, além do modo de colapso por perda de estabilidade global, esses também podem apresentar colapso por perda de estabilidade local; desta forma e se considerando os colmos de bambu como cilindros de paredes finas com altura L, espessura da parede h e raio a, onde $\mathrm{h}<<\mathrm{a}$, as forças e momentos internos que agem em um elemento de placa (Figura 1) são expressos em termos de forças e momentos por unidade de comprimento ao longo das arestas do elemento e podem ser expressos utilizando-se a Lei de Hooke e as relações cinemáticas, pela Eq. 9 (Bazant \& Cedolin, 1991):

$$
\begin{array}{ll}
\mathrm{N}_{\mathrm{x}}=\mathrm{C} \cdot\left(\varepsilon_{\mathrm{x}}+v \cdot \varepsilon_{\theta}\right) & \mathrm{M}_{\mathrm{x}}=\mathrm{D} \cdot\left(\kappa_{\mathrm{x}}+v \cdot \kappa_{\theta}\right) \\
\mathrm{N}_{\theta}=C \cdot\left(\varepsilon_{\theta}+v \cdot \varepsilon_{\mathrm{x}}\right) & \mathrm{M}_{\theta}=\mathrm{D} \cdot\left(\kappa_{\theta}+v \cdot \kappa_{\mathrm{x}}\right) \\
\mathrm{N}_{\mathrm{x} \theta}=\mathrm{C} \cdot \frac{1-v}{2} \cdot \gamma_{\mathrm{x} \theta} & \mathrm{M}_{\mathrm{x}}=\mathrm{D} \cdot(1-v) \cdot \kappa_{\mathrm{x} \theta}
\end{array}
$$

nas quais $\mathrm{C}$ e D são os parâmetros de rigidez axial e a flexão dados, respectivamente, pela Eq. 10:

$$
\mathrm{C}=\frac{\mathrm{E} \cdot \mathrm{h}}{1-v^{2}} \quad \text { e } \quad \mathrm{D}=\frac{\mathrm{E} \cdot \mathrm{h}^{3}}{12 \cdot\left(1-v^{2}\right)}
$$

Neste trabalho são utilizadas as hipóteses de Kirchhoff, dentre as quais se destacam: uma seção reta e normal ao plano médio da estrutura indeformada permanece reta e nor- mal ao plano na configuração deformada (desta forma, podese desprezar as deformações cisalhantes transversais) e as tensões normais na direção transversal são pequenas quando comparadas com outras componentes de tensão normal. Como consequência da primeira aproximação, as componentes de deslocamento em qualquer ponto da casca, u*, $\mathrm{v}^{*}, \mathrm{w}^{*}$, podem ser expressas em termos das quantidades correspondentes ao plano médio, u, v, w, através das equações Eq. 11, Eq. 12 e Eq. 13:

$$
\begin{aligned}
& \mathrm{u}^{*}=\mathrm{u}+\mathrm{z} \cdot \beta_{\mathrm{x}} \\
& \mathrm{v}^{*}=\mathrm{v}+\mathrm{z} \cdot \beta_{\mathrm{y}} \\
& \mathrm{w}^{*}=\mathrm{w}
\end{aligned}
$$

As componentes de deformação em qualquer ponto da casca $\varepsilon_{\mathrm{x}}, \varepsilon_{\theta}, \gamma_{\mathrm{x} \theta}$, podem ser expressas em termos dos deslocamentos correspondentes ao plano médio, por meio das equações Eq. 14, Eq. 15 e Eq. 16:

$$
\begin{aligned}
& \varepsilon_{\mathrm{x}}=\mathrm{u}_{, \mathrm{x}}+\frac{1}{2} \cdot \beta_{\mathrm{x}}^{2} \\
& \varepsilon_{\theta}=\left(\frac{\mathrm{v}_{\mathrm{,}}+\mathrm{w}}{\mathrm{a}}\right)+\frac{1}{2} \cdot \beta_{\theta}^{2} \\
& \gamma_{\mathrm{x} \theta}=\left(\frac{\mathrm{v}_{\mathrm{g}}}{\mathrm{a}}+\mathrm{v}_{\mathrm{x}}\right)+\frac{1}{2} \cdot \beta_{\theta} \cdot \beta_{\mathrm{x}}
\end{aligned}
$$

nas quais as rotações e curvaturas podem ser expressas pelas equações Eq. 17 a Eq. 21:

$$
\begin{aligned}
& \beta_{\mathrm{x}}=-\mathrm{w}_{, \mathrm{x}} \\
& \beta_{\theta}=\frac{-\mathrm{w}_{, \theta}}{\mathrm{a}} \\
& \kappa_{\mathrm{x}}=\beta_{\mathrm{x}, \mathrm{x}} \\
& \kappa_{\theta}=\beta_{\theta, \theta} \\
& \kappa_{\mathrm{x} \theta}=\frac{1}{2}\left(\frac{\beta_{\mathrm{x}, \theta}}{\mathrm{a}}+\beta_{\theta, \mathrm{x}}\right)
\end{aligned}
$$

A energia total do sistema pode ser expressa por meio da Eq. 22:

$$
\begin{aligned}
& \Pi=\frac{\mathrm{a} \cdot \mathrm{C}}{2} \cdot \iint\left(\varepsilon_{\mathrm{x}}^{2}+\varepsilon_{\theta}^{2}+2 \cdot v \cdot \varepsilon_{\mathrm{x}} \cdot \varepsilon_{\theta}+\frac{1-v}{2} \cdot \gamma_{\mathrm{x} \theta}^{2}\right) \cdot \mathrm{dx} \cdot \mathrm{d} \theta+ \\
& +\frac{\mathrm{a} \cdot \mathrm{D}}{2} \cdot \iint\left(\kappa_{\mathrm{x}}^{2}+\kappa_{\theta}^{2}+2 \cdot v \cdot \kappa_{\mathrm{x}} \cdot \kappa_{\theta}+2 \cdot(1-v) \cdot \kappa_{\mathrm{x} \theta}^{2}\right) \cdot \mathrm{dx} \cdot \mathrm{d} \theta+ \\
& +\iint \mathrm{p} \cdot \mathrm{u}_{, \mathrm{x}} \cdot \mathrm{dx} \cdot \mathrm{d} \theta
\end{aligned}
$$

na qual p é o carregamento axial por unidade de comprimento distribuído nos bordos do cilindro. Para problemas elásticos conservativos, o comportamento estático de um sistema depende apenas de sua energia potencial cuja estabilidade é garantida quando a energia potencial do mesmo apresenta um mínimo neste ponto; deste modo, uma configuração é estável se o incremento na energia potencial total devido a um campo qualquer de deslocamentos adicionais suficientemente pequenos e cinematicamente admissíveis é 


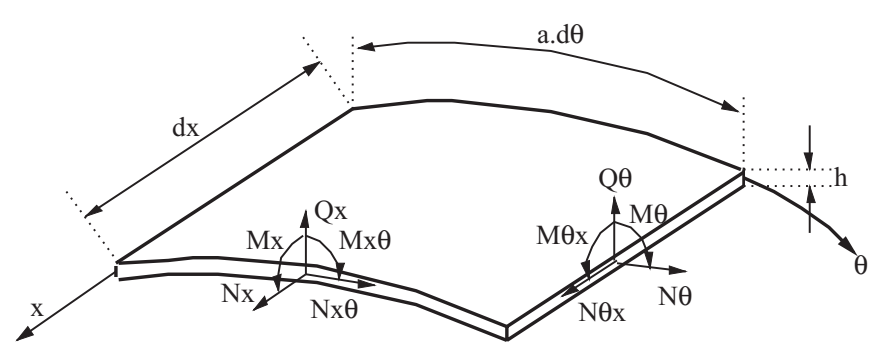

Figura 1. Elemento da casca cilíndrica

positivo definido. O equilíbrio é instável se o incremento é negativo para pelo menos uma configuração adjacente à configuração fundamental de equilíbrio; no caso de cilindros de paredes finas, além do modo de colapso por perda de estabilidade global, esses também podem apresentar colapso por perda de estabilidade local; desta forma e se considerando os colmos de bambu como cilindros de paredes finas com altura L, espessura da parede $\mathrm{h}$ e raio a, onde $\mathrm{h}<<\mathrm{a}$, as forças e momentos internos que agem em um elemento de placa (Figura 1) são expressos em termos de forças e momentos por unidade de comprimento ao longo das arestas do elemento e podem ser expressos utilizandose a Lei de Hooke e as relações cinemáticas, pela Eq. 9 (Bazant \& Cedolin, 1991). Expandindo a energia potencial em série de Taylor, tem-se:

$$
\Delta \Pi=\frac{1}{2 !} \delta^{2} \Pi+\frac{1}{3 !} \delta^{3} \Pi+\frac{1}{4 !} \delta^{4} \Pi
$$

na qual para o sistema ser estável $\Delta \pi>0$ e como o primeiro termo da Eq. 23 é dominante, a condição de estabilidade do equilíbrio é determinada pela segunda variação. O sistema será estável se a segunda variação $\delta^{2} \Pi$ for positiva. O caso crítico ocorre quando a segunda variação se anula para um ou mais campos de deslocamentos incrementais linearmente independentes, ou seja, $\delta\left[\delta^{2} \Pi\right]=0$.

Para o cálculo das cargas e modos críticos utilizou-se a formulação apresentada; assim, substituindo-se a Eq. 22 na Eq. 23 e se igualando a segunda variação da energia a zero, tem-se a Eq. 24:

$$
\begin{aligned}
& \nabla^{4} \mathrm{u} 1=-\frac{\mathrm{n}}{\mathrm{a}} \cdot \mathrm{w}_{1, x x x x}+\frac{1}{\mathrm{a}^{3}} \cdot \mathrm{w}_{1, x \theta \theta} \\
& \nabla^{4} \mathrm{v} 1=-\frac{2+\mathrm{v}}{\mathrm{a}^{2}} \cdot \mathrm{w}_{1, x x \theta}-\frac{1}{\mathrm{a}^{4}} \cdot \mathrm{w}_{1, \theta \theta \theta} \\
& D \nabla^{8} \mathrm{w} 1+\frac{1-\mathrm{v}^{2}}{\mathrm{a}^{2}} \cdot \mathrm{C} \cdot \mathrm{w} 1_{, x x x x}-\nabla^{4}\left(\mathrm{~N}_{\mathrm{x} 0} \cdot \mathrm{w} 1_{, x x}+\frac{2}{\mathrm{a}} \cdot \mathrm{N}_{\mathrm{x} \theta 0} \mathrm{~W} 1_{x \theta}+\frac{1}{\mathrm{a}^{2}} \cdot \mathrm{N}_{\theta 0} \cdot \mathrm{w} 1_{, \theta \theta}\right)=0
\end{aligned}
$$

na qual as condições de contorno são expressas pela Eq. 25:

$$
\mathrm{N}_{\mathrm{x} \theta}=-\frac{\mathrm{P}}{2 \cdot \pi \cdot \mathrm{a}}, \quad \mathrm{N}_{\mathrm{x} \theta 0}=0, \quad \mathrm{e}, \quad \mathrm{N}_{\theta 0}=0
$$

Resolvendo-se o sistema de equações Eq. 24 juntamente com a Eq. 25, obtém-se que a equação da carga crítica das cascas cilíndricas submetidas a carregamento axial é expressa pela Eq. 26:

$$
P=\frac{\left(\left(\frac{m \cdot \pi \cdot a}{L}\right)^{2}+n^{2}\right)^{2}}{\left(\frac{m \cdot \pi \cdot a}{L}\right)^{2}} \cdot \frac{D}{a^{2}}+\frac{\left(\frac{m \cdot \pi \cdot a}{L}\right)^{2}}{\left(\left(\frac{m \cdot \pi \cdot a}{L}\right)^{2}+n^{2}\right)^{2}} \cdot\left(1-v^{2}\right) \cdot C
$$

Para cada valor de m e $\mathrm{n}$ tem-se uma carga e um modo crítico de flambagem correspondente. Deste modo, para um dado $m$ existe um valor de $n$ que minimiza $P$, e assim foi desenvolvida uma sub-rotina em linguagem $C$, que calcula, para m, os valores n que minimizam Eq. 26.

\section{Materiais}

O agregado miúdo utilizado foi areia quartzosa com módulo de finura de 2,11, diâmetro máximo de 2,4 mm e massa específica aparente de 1,62 $\mathrm{kg} \mathrm{dm}^{-3}$ (ABNT, 2003a; 2003b). Utilizou-se agregado graúdo de origem basáltica com módulo de finura de 6,8, diâmetro máximo de $19 \mathrm{~mm}$ e massa específica aparente de 1,92 $\mathrm{kg} \mathrm{dm}^{-3}$ (ABNT, 2003a; 2003c). O cimento utilizado foi o de alta resistência inicial CP-V. Definiram-se as porcentagens dos materiais a partir de uma curva experimental de dosagem para a qual se adotou um teor de argamassa de 51\%, uma relação água/materiais secos de $9 \%$ e abatimento no cone de Abrams de $20 \pm 2 \mathrm{~cm}$. As resistências dos concretos foram avaliadas aos sete dias, por meio de ensaio de corpos-de-prova cilíndricos 15 x $30 \mathrm{~cm}$. O traço obtido foi 1:2,86:3,7, fator água/cimento de 0,68 e abatimento de tronco de cone de $20 \mathrm{~cm}$. A resistência média obtida com o respectivo desvio-padrão foi 23,54 e 2,07 MPa, respectivamente.

O bambu empregado foi da espécie Dendrocalamus giganteus, cujos colmos foram cortados com idades entre quatro e cinco anos e deixados secar à sombra, em temperatura ambiente, pelo período de três meses e apresentavam comprimento médio de $20 \mathrm{~m}$, foram divididos em três trechos de

\begin{tabular}{|c|c|c|c|c|c|c|c|c|}
\hline Região & $\begin{array}{c}f_{b t} \\
(\mathrm{MPa})\end{array}$ & $\begin{array}{c}E_{b t} \\
(\mathrm{GPa})\end{array}$ & $\begin{array}{c}f_{b} \\
\left(M^{b P a}\right.\end{array}$ & $\begin{array}{c}E_{b c} \\
(\mathrm{GPa})\end{array}$ & $v$ & $\begin{array}{c}\tau_{\mathrm{b}} \\
(\mathrm{MPa})\end{array}$ & $\begin{array}{c}s_{n} \\
(\mathbf{c m})\end{array}$ & $\begin{array}{c}\phi_{\mathrm{e}} \\
(\mathrm{cm})\end{array}$ \\
\hline Nodal & 97 & 13 & 58 & 19 & - & - & 1 & 1 \\
\hline Internoidal & 277 & 23 & 58 & 22 & 0,24 & 7,8 & 48,3 & 15,2 \\
\hline
\end{tabular}
igual comprimento, aqui denominados: basal, intermediário e topo; apenas os trechos basais e intermediários foram utilizados. As propriedades mecânicas do bambu utilizado são apresentadas na Tabela 1.

Tabela 1. Propriedades mecânicas do bambu Dendrocalamus giganteus

Nota: $\mathrm{f}_{\mathrm{tb}}$ é a resistência a tração do bambu, $\mathrm{E}_{\mathrm{bt}}$ é 0 módulo de elasticidade à tração do bambu, $\mathrm{f}_{\mathrm{bc}}$ é a resistência à compressão do bambu, $E_{b c}$ é o módulo de elasticidade à compressão do bambu, $v$ é $o$ coeficiente de Poisson, $\tau_{b}$ é a resistência ao cisalhamento e paralelo às fibras do bambu, $S_{n}$ é 0 espaçamento entre nós do bambu e, finalmente, $\phi_{\mathrm{e}}$ é 0 diâmetro externo do bambu

\section{Característica dos espécimes}

Foram ensaiados 18 pilares de bambu, dos quais nove se constituíam de colmos de bambu preenchidos com concreto e nove formados apenas por colmos de bambu. Os pilares apresentaram altura 1, 1,5 e $2 \mathrm{~m}$, em triplicadas. As alturas dos pilares foram calculadas de modo que a força relativa à resistência mecânica dos colmos correspondesse a, no máximo, um quarto da carga de flambagem calculada por meio da equação de Euler, Eq. 8 que, para os pilares de 2 m, foi da ordem de $650 \mathrm{kN}$. 


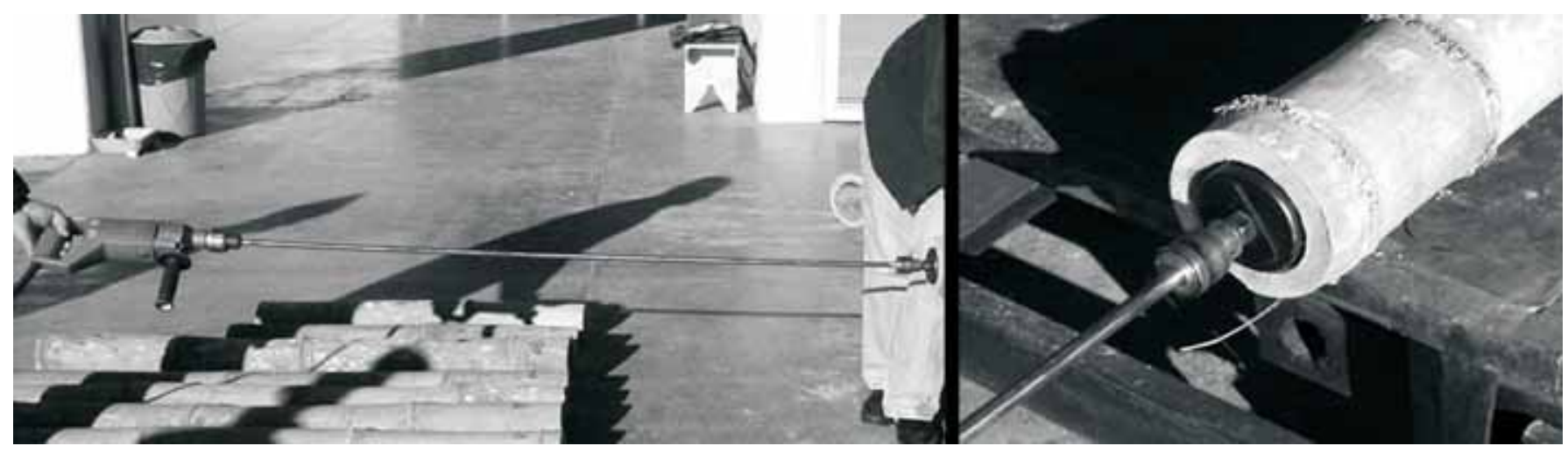

Figura 2. Preparação dos colmos de bambu

Inicialmente, selecionaram-se os colmos de bambu de modo a apresentar eixo retilíneo, os quais foram cortados em serra circular nos comprimentos predefinidos; após o corte, os diafragmas dos colmos foram retirados por meio de uma serra copo com $10 \mathrm{~cm}$ de diâmetro adaptada a um eixo de dois metros e conectada a uma furadeira elétrica (Figura 2); em seguida, os colmos foram limpos e suas dimensões medidas com paquímetro e trena.

Todos os colmos foram engastados em blocos de concre- to armado com dimensões de $20 \times 30$ x $30 \mathrm{~cm}$. As formas dos blocos foram confeccionadas em madeira laminada compensada e os blocos armados com barras de aço com diâmetro de $5 \mathrm{~mm}$ espaçadas a cada $14 \mathrm{~cm}$ nas duas direções e por três estribos de aço, com o mesmo diâmetro, espaçados a cada $9 \mathrm{~cm}$. Os colmos de bambu foram ancorados aos blocos por 4 barras de $5 \mathrm{~mm}$. Os detalhes do bloco e da ancoragem são apresentados na Figura 3; após a limpeza, os colmos foram fixados em um gabarito para garantir o alinhamento verti-

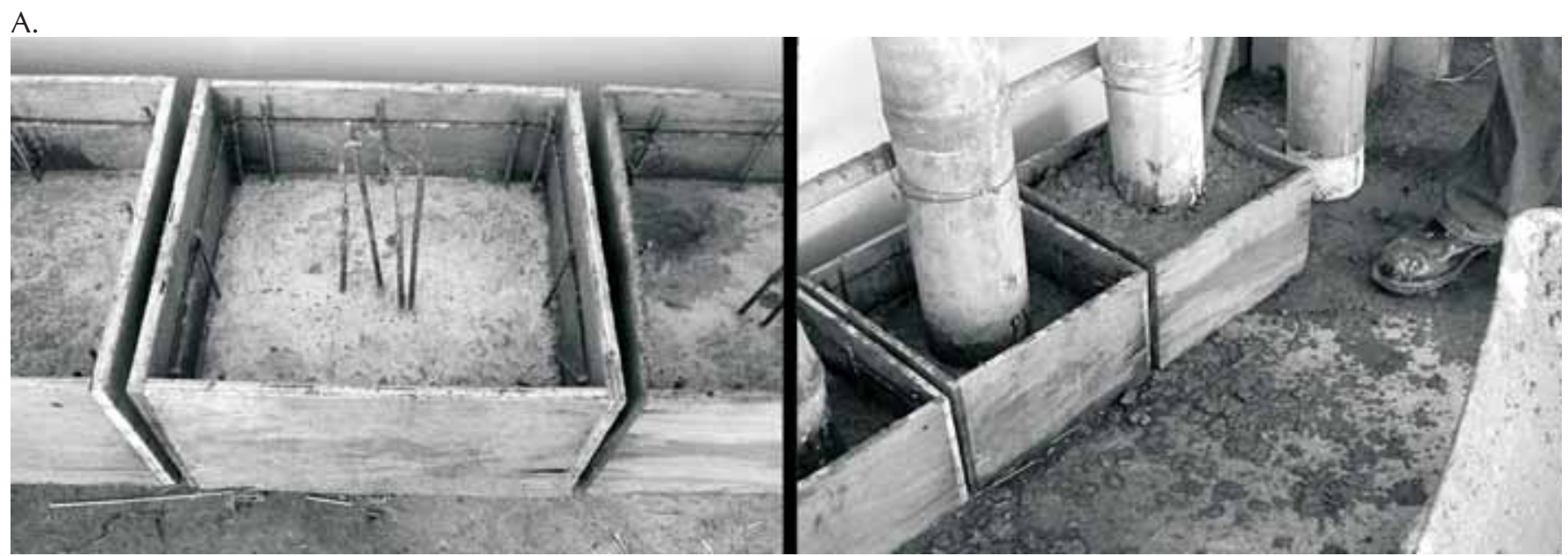

B.

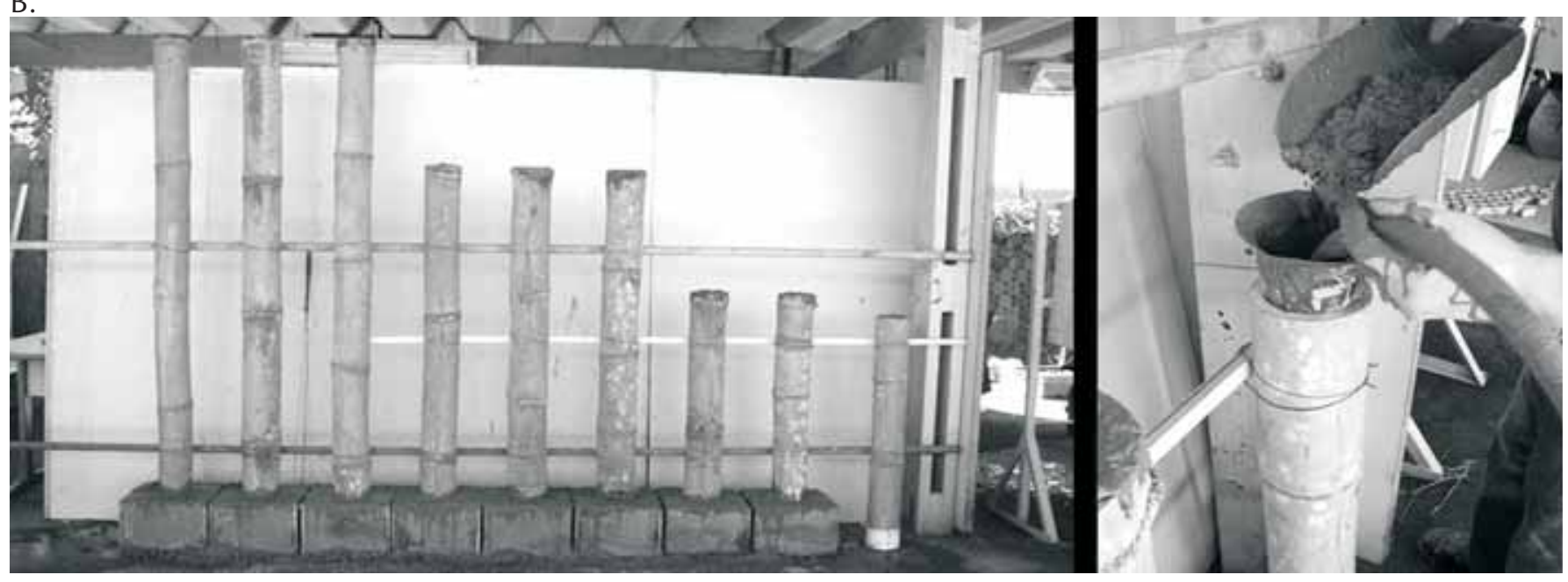

Figura 3. Concretagem dos blocos de engastamento (A) e dos pilares (B) 
cal, com suas bases dentro das formas dos blocos de concreto; em seguida ao posicionamento dos colmos, procedeu-se à concretagem dos blocos com auxílio de um vibrador de imersão. Três corpos-de-prova cilíndricos 15 x 30 cm de concreto foram moldados para posterior análise da resistência a compressão. Realizou-se a cura dos blocos de concreto armado nas próprias formas dispondo-se espumas umidificadas sobre os mesmos. Evitou-se a molhagem direta dos blocos de modo a não permitir absorção elevada de água do bambu e uma possível variação dimensional, o que poderia prejudicar o engastamento do colmo ao bloco. O procedimento de cura dos blocos foi iniciado 24 h após de concretagem.

Decorridos 7 dias da concretagem dos blocos, procedeuse à concretagem dos colmos de bambu. Nove colmos de bambu, com comprimento de 1, 1,5 e 2 m, sendo três de cada comprimento, foram preenchidos com concreto, o qual foi lançado pela região superior dos colmos (Figura 3); novamente, três corpos-de-prova cilíndricos 15 x $30 \mathrm{~cm}$ foram moldados para posterior análise da resistência a compressão do concreto. O procedimento de cura foi similar ao dos blocos.

\section{Metodologia de ensaio}

Em referência aos pilares, foram ensaiados com controle de força em prensa hidráulica cuja capacidade era de $5000 \mathrm{kN}$ e vão livre de ensaio de $4 \mathrm{~m}$ e o carregamento aplicado de modo quase estático, a uma taxa média de aplicação da força de $5 \mathrm{kN} \mathrm{min}{ }^{-1}$; por sua vez, os pilares foram posicionados na prensa, através de um gabarito; em seguida, uma rótula tri-dimensional foi colada na parte superior dos pilares com resina epoxídica.

Dois anéis de aço foram posicionados ao longo da altura dos pilares para servirem de suporte aos três medidores de deslocamento mecânicos com precisão de $0,01 \mathrm{~mm}$, os quais foram dispostos equidistantes, conforme os vértices de um triângulo equilátero. O comprimento de avaliação dos deslocamentos para os pilares de 1, 1,5 e $2 \mathrm{~m}$ foi de 60 , 90 e $120 \mathrm{~cm}$, respectivamente. As leituras dos medidores de deslocamento foram realizadas manualmente a cada $5 \mathrm{kN}$ e o ensaio finalizado quando do colapso do pilar. A Figura 4 apresenta detalhes dos ensaios dos pilares.

\section{RESULTADOS E DISCUSSÃO}

\section{Comportamento geral}

O concreto dos blocos e dos pilares apresentou resistência em concordância com o dimensionado, com valor de 20,12 MPa. A metodologia de ensaio mostrou-se adequada, sendo possível obter as curvas força vs. deformação para todos os pilares ensaiados; enquanto os pilares de bambu sem concreto apresentaram colapso caracterizado pelo surgimento de fissuras longitudinais, paralelas às fibras do bambu, iniciadas nas regiões internodais por forte efeito de flambagem, as quais se propagavam ao longo do comprimento dos pilares, formando tiras esbeltas (Figura 5A), com perda brusca da capacidade de carga, não sendo possível a leitura de deslocamentos a partir daí. O colapso dos pilares de bambu preenchidos com concreto se iniciava com o surgimento prematuro

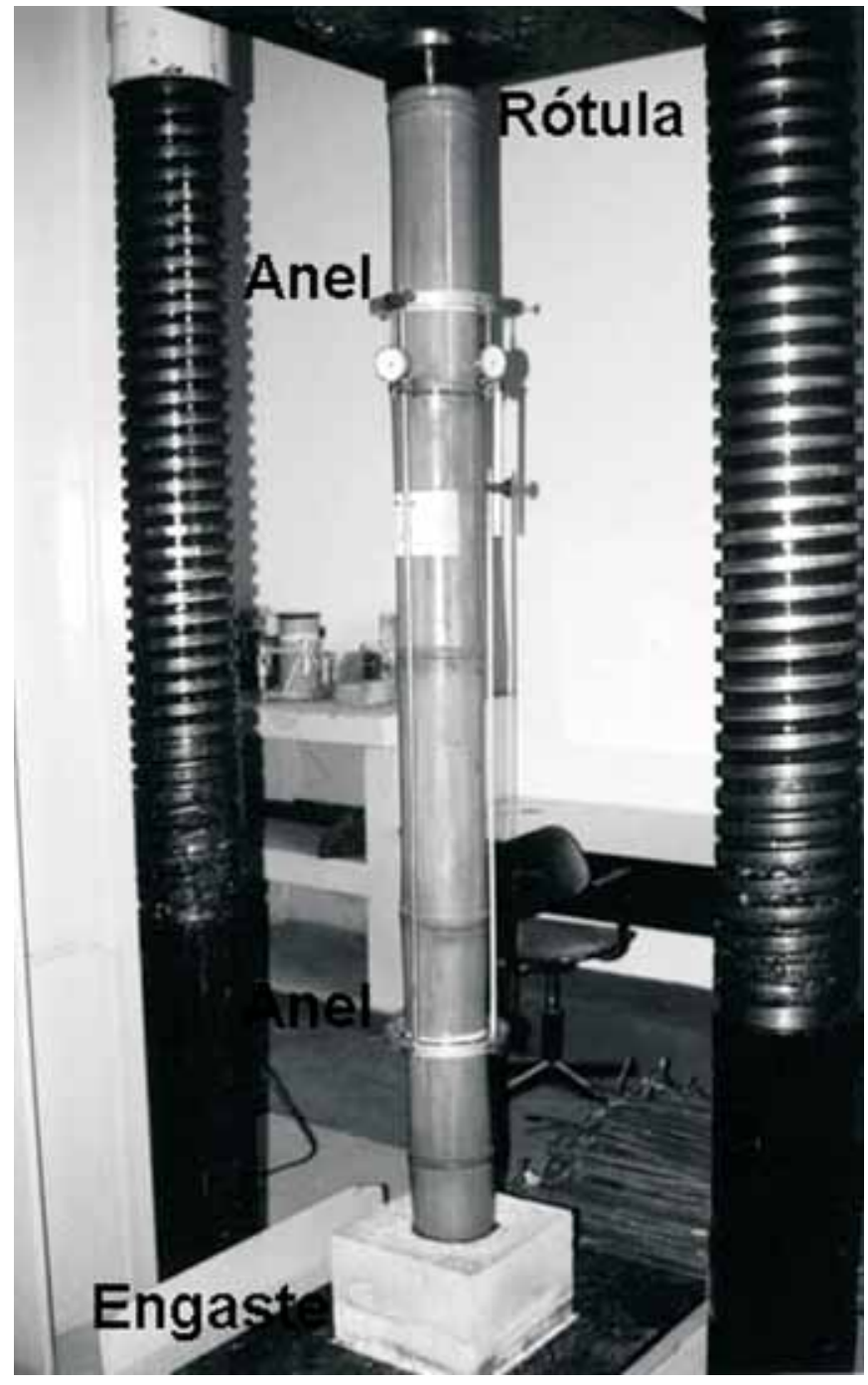

Figura 4. M étodo de ensaio

A.

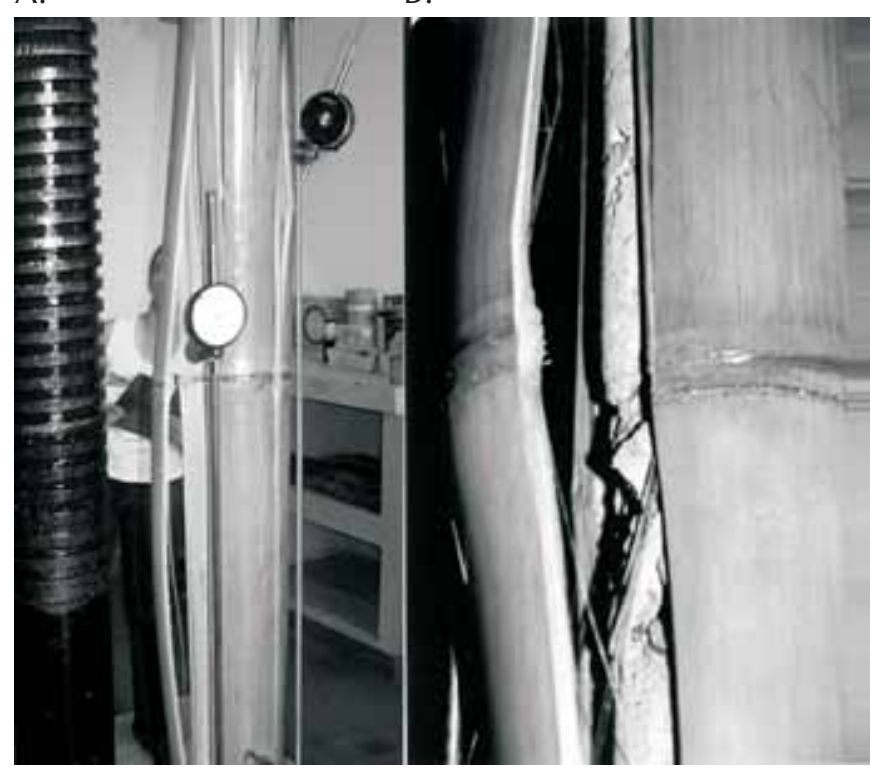

Figura 5. Detalhes do modo de falha dos pilares de bambu: pilares sem concreto $(A)$ e pilares com concreto $(B)$ 
de fissuras na região nodal, que se propagavam pelos trechos internodais. Analisando-se visualmente esses pilares após o ensaio, verificou-se uma expansão lateral com esmagamento do concreto na região dos nós, ocasionado pela menor seção transversal de concreto com provável resistência reduzida. O diâmetro interno dos colmos na posição dos nós apresentou seção transversal inferior à do trecho internodal, com estricção brusca, limitada ao diâmetro da serra copo para a retirada dos diafragmas; também na região dos nós o concreto indicou característica porosa, ocasionada pela maior absorção da água dos poros expostos do bambu pela retirada dos diafragmas com serra copo, com possível redução em sua resistência. Apresentam-se, na Figura 5B, detalhes do modo de falha dos pilares de bambu e os preenchidos com concreto.

\section{Comportamento mecânico dos pilares}

Apresentam-se, na Figura 6, as curvas teóricas e experimentais força vs. deformação dos pilares ensaiados e a curva de tendência calculada por meio de aproximação dos mínimos quadrados, em que para todos os pilares formados apenas por

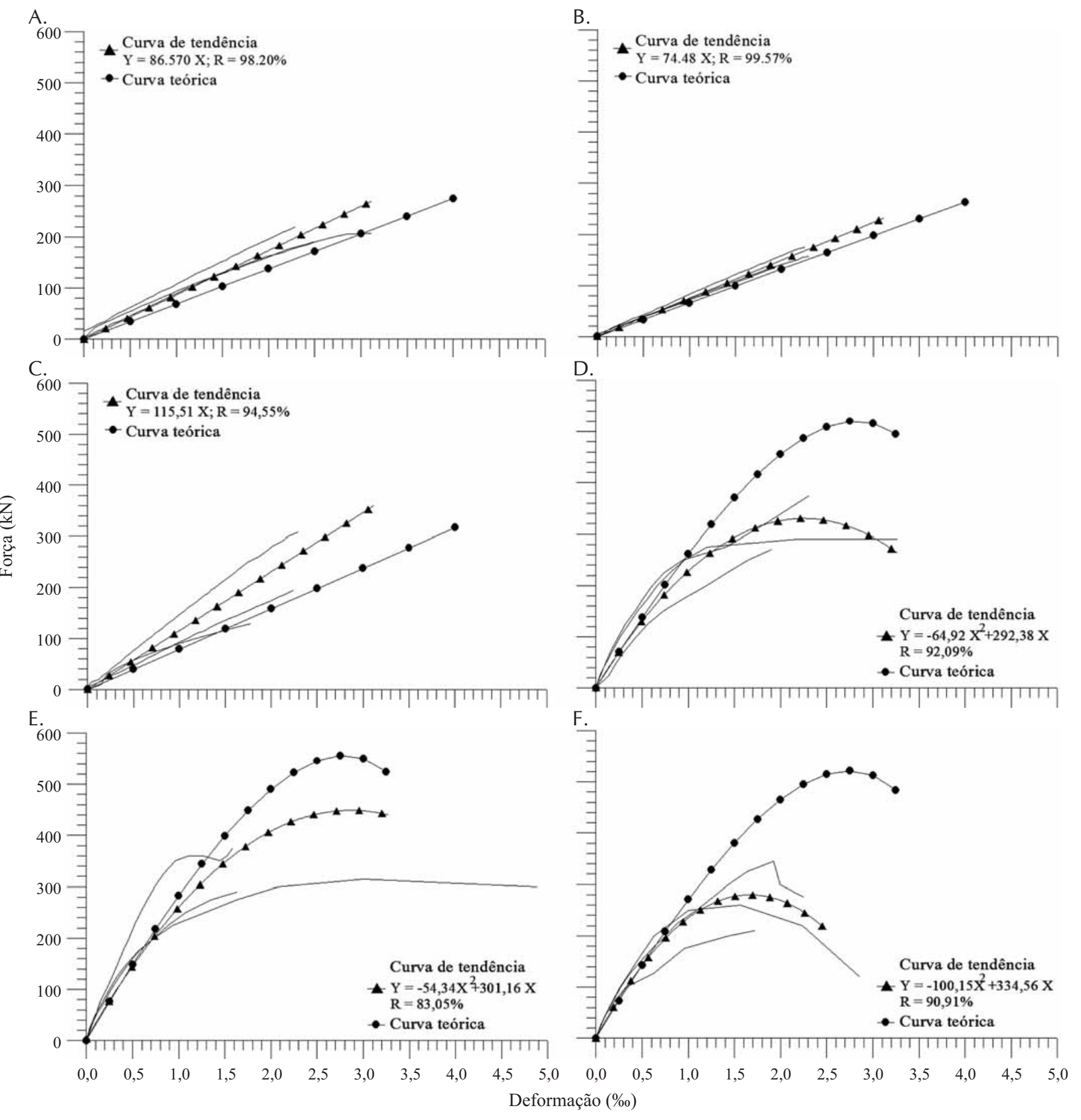

Figura 6. Curvas teóricas e experimentais força vs. deformação dos pilares ensaiados: A) pilares de $1 \mathrm{~m}$ sem concreto; B) pilares de 1,5 $\mathrm{m}$ sem concreto; C) pilares de $2 \mathrm{~m}$ sem concreto; D) pilares de $1 \mathrm{~m}$ com concreto; E) pilares de 1,5 m com concreto, e F) pilares de $2 \mathrm{~m}$ com concreto 
colmos de bambu, o comportamento mecânico foi elástico linear, sendo bastante similar entre os pilares com mesmas características físicas. Para as três alturas, observaram-se coeficientes de correlação, $\mathrm{R}^{2}$, superiores a 94\%. Comparando-se as curvas teóricas e as experimentais, verifica-se que a Eq. 1 representou, com boa aproximação, o comportamento experimental e, em todos os casos, a rigidez experimental foi superior à teórica, constatada pela inclinação das curvas. Não se observaram reduções de rigidez para os pilares com maior altura, fato este ocasionado pelo elevado índice de rigidez dos elementos ensaiados; já para os pilares de bambu preenchidos com concreto (Figura 6D, E, F), constatam-se maiores discrepâncias entre o comportamento teórico e o experimental, principalmente para deformações superiores a 1\%o, quando se inicia o comportamento não linear desses elementos; como já mencionado, este fato pode ter sido ocasionado pela estricção da seção transversal do concreto e sua menor resistência nas regiões nodais. O comportamento mecânico entre as réplicas dos pilares preenchidos com concreto e com mesmas características físicas foi similar; todavia, o coeficiente de correlação, $\mathrm{R}^{2}$, foi inferior ao obtido para os pilares de bambu sem concreto, variando entre 83 e $92 \%$.

Tem-se, na Tabela 2, os valores das forças últimas experimentais e teóricas calculadas com base nas equações Eq. 1, 8 e 26 para todos os pilares ensaiados. Para os pilares de bambu sem preenchimento de concreto, as forças correspondentes à flambagem global obtidas com a Eq. 8 apresentaram valores, em média, 20 vezes superiores à experimental; já para as cargas de flambagem local, calculadas pela Eq. 26, esta correlação foi, em média, 3 vezes superior. Finalmente, observa-se que o valor da força teórica calculada pela resistência dos materiais, Eq. 1, coincide com o valor experimental diferindo apenas em 5\%. Diante do exposto, pode-se afirmar que para os pilares de bambu sem concreto o colapso ocorreu por ruptura do bambu, seguindo pela flambagem local das tiras de bambu.

Os pilares preenchidos com concreto apresentaram forças últimas experimentais discrepantes das teóricas. As forças teóricas de flambagem global e local foram superiores às experimentais em 20 e 10 vezes, respectivamente; já a força última, calculada de acordo com a resistência dos materiais, diferiu cerca de 2 vezes do valor experimental, que pode ter sido ocasionado pela menor resistência do concreto na região nodal e pela redução da seção transversal de concreto nos nós. Observou-se, durante os ensaios, que esses pilares entraram em colapso pela ruptura do concreto na região nodal seguida pela flambagem local das tiras de bambu.

Todos os pilares foram projetados para apresentar colapso por ruptura dos materiais; contudo, a possibilidade de acoplamento entre os modos de colapso por flambagem local e por ruptura dos materiais, foi observada em alguns pilares.

Confrontando-se as forças experimentais dos pilares de bambu com e sem concreto, verifica-se que a adição do concreto não gerou resultados satisfatórios sendo, deste modo, mais eficaz e econômica a utilização apenas dos colmos de bambu. Verificou-se, enfim, que a extração dos diafragmas dos colmos para os pilares de bambu sem preenchimento por concreto, colaborou para a flambagem local das tiras de bambu após a força última (Figura 5).

Com base na relação entre força teórica e força experimental (apresentadas na Tabela 2) e o índice de esbeltez dos pilares (relação entre o comprimento e o raio de giração), realizaram-se análises de correlação simples entre variáveis para os pilares com e sem concreto. Observou-se que não existe correlação entre os índices de esbeltez estudados e as forças últimas dos pilares, para nível de confiabilidade de 95\%, tanto para os pilares com concreto quanto para os sem concreto.

Tabela 2. Forças últimas teóricas e experimentais dos pilares

\begin{tabular}{|c|c|c|c|c|c|c|c|c|c|c|}
\hline \multirow{2}{*}{ Pilar } & \multirow{2}{*}{$L(m)$} & \multicolumn{2}{|c|}{ Preenchimento } & \multirow{2}{*}{$\begin{array}{l}P_{\text {exp }}(k N) \\
\text { (1) }\end{array}$} & \multirow{2}{*}{$\begin{array}{c}P_{\text {teo }}(\text { Eq.1) }(\mathrm{kN}) \\
\text { (2) }\end{array}$} & \multirow{2}{*}{$\begin{array}{c}P_{\text {teo }}(E q .8)(k N) \\
\text { (3) }\end{array}$} & \multirow{2}{*}{$\begin{array}{c}P_{\text {teo }}(\mathrm{Eq} .15)(\mathrm{kN}) \\
\text { (4) }\end{array}$} & \multirow{2}{*}{$\frac{(2)}{(1)}$} & \multirow{2}{*}{$\frac{(3)}{(1)}$} & \multirow{2}{*}{$\frac{(4)}{(1)}$} \\
\hline & & Com & Sem & & & & & & & \\
\hline$P_{1}$ & 1,0 & & $\mathrm{X}$ & 193,50 & 183,21 & 3384,28 & 520,20 & 0,95 & 17,49 & 2,69 \\
\hline $\mathrm{P}_{2}$ & 1,0 & & $x$ & 219,00 & 197,13 & 4409,42 & 477,60 & 0,90 & 20,13 & 2,18 \\
\hline $\mathrm{P}_{3}$ & 1,0 & & $x$ & 207,00 & 183,21 & 3624,51 & 510,90 & 0,89 & 17,51 & 2,47 \\
\hline $\mathrm{P}_{4}$ & 1,5 & & $x$ & 180,00 & 180,12 & 3365,93 & 520,40 & 1,00 & 18,70 & 2,89 \\
\hline$P_{5}$ & 1,5 & & $x$ & 157,00 & 180,12 & 3365,93 & 520,40 & 1,15 & 21,44 & 3,31 \\
\hline $\mathrm{P}_{6}$ & 1,5 & & $x$ & 139,00 & 180,12 & 3365,93 & 520,40 & 1,30 & 24,22 & 3,74 \\
\hline $\mathrm{P}_{7}$ & 2,0 & & $x$ & 198,00 & 180,12 & 3365,93 & 520,40 & 0,91 & 17,00 & 2,63 \\
\hline $\mathrm{P}_{8}$ & 2,0 & & $x$ & 308,00 & 296,22 & 6678,21 & 1069,00 & 0,96 & 21,68 & 3,47 \\
\hline \multirow[t]{2}{*}{$\mathrm{P}_{9}$} & 2,0 & & $x$ & 129,00 & 174,77 & 3075,64 & 540,10 & 1,35 & 23,84 & 4,19 \\
\hline & & & & \multicolumn{4}{|c|}{ Média - Coeficiente de Variação (\%) } & $1,05-16,93$ & $20,22-13,57$ & $3,06-21,42$ \\
\hline$P_{10}$ & 1,0 & $\mathrm{X}$ & & 290,00 & 670,39 & 12010,77 & 4858,00 & 2,31 & 41,42 & 16,75 \\
\hline$P_{11}$ & 1,0 & $\mathrm{x}$ & & 375,00 & 531,87 & 11843,15 & 1543,00 & 1,42 & 31,58 & 4,11 \\
\hline $\mathrm{P}_{12}$ & 1,0 & $\mathrm{x}$ & & 270,00 & 484,11 & 6415,08 & 2255,00 & 1,79 & 23,76 & 8,35 \\
\hline $\mathrm{P}_{13}$ & 1,5 & $\mathrm{x}$ & & 300,00 & 604,27 & 5208,10 & 2597,00 & 2,01 & 17,36 & 8,66 \\
\hline $\mathrm{P}_{14}$ & 1,5 & $x$ & & 290,00 & 470,21 & 4053,41 & 1287,00 & 1,62 & 13,98 & 4,44 \\
\hline $\mathrm{P}_{15}$ & 1,5 & $\mathrm{x}$ & & 375,00 & 732,05 & 6286,88 & 4285,00 & 1,95 & 16,77 & 11,43 \\
\hline $\mathrm{P}_{16}$ & 2,0 & $x$ & & 250,00 & 441,83 & 1869,90 & 1334,00 & 1,77 & 7,48 & 5,34 \\
\hline $\mathrm{P}_{17}$ & 2,0 & $x$ & & 275,00 & 678,65 & 3263,65 & 3247,00 & 2,47 & 11,87 & 11,81 \\
\hline \multirow[t]{2}{*}{$\mathrm{P}_{18}$} & 2,0 & $x$ & & 120,00 & 584,58 & 2746,64 & 2298,00 & 4,87 & 22,89 & 19,15 \\
\hline & & & & \multicolumn{4}{|c|}{ Média - Coeficiente de Variação (\%) } & $2,25-46,15$ & $20,79-50,63$ & $10,00-53,18$ \\
\hline
\end{tabular}




\section{CONCLUSÕES}

1. A influência do concreto nos pilares foi pequena, sendo o comportamento estrutural teórico vs. experimental dos pilares compostos bambu-concreto bastante discrepante.

2. A extração dos diafragmas dos colmos dos pilares de bambu foi inadequada frente aos efeitos de flambagem das tiras de bambu formadas após o colapso.

3. Todos os pilares romperam por ruptura dos materiais, seguida pela flambagem local das tiras de bambu formadas após a força última.

4. Não foi observada correlação entre os índices de esbeltez e as forças últimas.

5. O comportamento dos pilares de bambu sem preenchimento de concreto mostrou-se mais viável estruturalmente, em relação aos preenchidos com concreto.

\section{AGRADECIMENTOS}

Ao CNPq, pela concessão de bolsa de estudo para os três últimos autores, e à FACEPE, pelo auxílio à pesquisa. Finalmente, os autores são gratos à Hidrelétrica de Itaipu, por haver cedido seu Laboratório de Tecnologia do Concreto, para execução dos ensaios.

\section{LITERATURA CITADA}

ABNT - Associação Brasileira de Normas Técnicas. NBRNM248 - Agregados: Determinação da composição granulométrica. Rio de Janeiro: ABNT, 2003a. 7p.

ABNT - Associação Brasileira de Normas Técnicas. NBRNM52 - Agregado miúdo - Determinação de massa específica e massa específica aparente. Rio de Janeiro: ABNT, 2003b. 7p.
ABNT - Associação Brasileira de Normas Técnicas. NBRNM53 - Agregado graúdo - Determinação de massa específica, massa específica aparente e absorção de água. Rio de Janeiro: ABNT, 2003c. 7p.

Albermani, F.; Goh, G. Y.; Chan, S. L. Lightweight bamboo double layer grid system. Engineering Structures, v.29, p.1499-1506, 2007.

Bazant, Z. P.; Cedolin, L. Stability of structures, elastic, inelastic, fracture and damage theories. New York: Oxford University Press, 1991. 580p.

Beraldo A. L.; Rivero, L. A. Bambu laminado coldado (BLC). Floresta e Ambiente, v.10, n.2, p.36-46, 2003.

Chung, K. F.; Yu, W. K. Mechanical properties of structural bamboo for bamboo scaffoldings. Engineering Structures, v.24, p.429-442, 2002.

Lima Jr., H. C.; Dias, A. A. Vigas mistas de madeira de reflorestamento e bambu laminado colado: análise teórica e experimental. Revista Brasileira de Engenharia Agrícola e Ambiental, v.5, n.3, p.519-524, 2001.

Lima Jr., H. C.; Willrich, F. L.; Barbosa, N. P.; Rosa, M. A.; Cunha, B. S. Durability analysis of bamboo as concrete reinforcement. Materials and Structures, v.41, p.805-999, 2008.

Lima Júnior, H. C.; Willrich, F. L.; Fabro G. Vigas de concreto reforçadas com bambu Dendrocalamus giganteus II: Modelagem e critérios de dimensionamento. Revista Brasileira de Engenharia Agrícola e Ambiental, v.9, n.4, p.652-659, 2005.

Riaño, N. M.; Londoño, X.; López, Y.; Gómez, J. H. Plant growth and biomass distribution on Guadua angustifolia Kunth in relation to ageing in the Valle del Cauca - Colombia. The Journal of the American Bamboo Society, v.1, n.16, p.43-51, 2002.

Yu, W. K.; Chung, K. F.; Chan, S. L. Column buckling of structural bamboo. Engineering Structures, v.25, p.755-768, 2003.

Yu, W. K.; Chung, K. F.; Chan, S. L. Axial buckling of bamboo columns in bamboo scaffolds. Engineering Structures, v.27, p.61-73, 2005. 